\title{
Health Impacts of Global Climate Change
}

\author{
Mitsuru Ando ${ }^{1}$ and Warren T. Piver ${ }^{2}$
}

\begin{abstract}
Increased combustion of fossil fuels, along with many industrial and agricultural activities are resulting in greater emissions of greenhouse gases such as carbon dioxide, methane and nitrous oxide into the atmosphere. The continuing increased emissions of these greenhouse gases is contributing to global warming and climate change because of the greenhouse effect. Global warming may become a critical issue for increased frequency and severity of heat stress morbidity and mortality in summer, increased exposure to air pollution, and the spread of vectorborne and water-borne infectious diseases from tropical latitudes to more northern and southern latitudes and higher elevations.

In this symposium, four presentations will discuss the possible impacts of global warming on a higher incidence of heat stress related mortality and morbidity, expansion of regions for vectorborne and water-borne diseases, contribution of higher temperatures and air pollution to mortality, and the possible relationship of El Nino-Southern Oscillation to the spread of malaria to more southern latitudes and higher elevations. J Epidemiol,1996; 6 : S159-S160.
\end{abstract}

global warming, heat stress, air pollution, malaria vector

\section{SUMMARY OF PRESENTATIONS}

Increased human activities such as greater combustion of fossil fuels as energy resources, expanded industrial and agricultural activities and changes in land-use, are contributing to global climate change. These activities are resulting in greater emissions of carbon dioxide into the atmosphere. Because carbon dioxide is a greenhouse gas, increasing atmospheric concentrations result in higher temperatures because of the greenhouse effect. Using global climate change models that simulate interactions between atmospheric and oceanic circulation, Working Group I of the Intergovernmental Panel on Climate Change (IPCC) estimated in a 1995 report that surface temperatures will rise 1 to $3.5^{\circ} \mathrm{C}$ during the next 100 years because of a doubling of atmospheric concentrations of carbon dioxide. As a result, many human communities and natural ecosystems will be adversely affected. To assess the impacts of temperature changes of this magnitude, Working Group II of the IPCC was convened. One of the tasks of Working Group II was to assess the impacts of climate change on human health. At the same time, a Task Group was convened by WHO, WMO and UNEP to examine the impacts of climate change on human health in much greater detail than could be done in the report for Working Group II. In this symposium, four presentations will discuss several important direct and indirect impacts on human health of climate change that were discussed in these two reports.

(1) For human health, an important direct effect of climate change could be a greater frequency of heat waves in summer resulting in a higher incidence of heat stress related mortality and morbidity. In temperate large cities, an increase in extreme heat stress related morbidity and mortality would be associated with global warming and an enhanced heat island effect. In particular, heat stress related diseases such as heat stroke, would show a dramatic increase in the elderly as a result of very hot summer temperatures. To evaluate the health risk of extreme heat stress in hot summers, epidemiological studies were caried out in Tokyo, Japan and in Nanjing and Wuhan, China. In Tokyo for July and August of 1993 and 1994, the total number of emergency cases was 130,560. For Tokyo,

\footnotetext{
${ }^{1}$ National Institute for Environmental Studies, Tsukuba, Japan

${ }^{2}$ National Institute of Environmental Health Sciences, NC, U.S.A.

Address for correspondence : Mitsuru Ando, National Institute of Environmental Studies, 16-2 Onogawa Tsukuba, 305 Japan.
} 
Nanjing and Wuhan, a significant positive correlation was observed between maximum or mean daily temperature and the daily number of heatstroke patients above a certain threshold temperature. A significant positive correlaion was observed between the temperature and the number of emergency patients. The regression analysis showed that the number of heat stroke patients increased exponentially when the mean daily temperature and maximum daily temperature exceeded $27^{\circ} \mathrm{C}$ and $31^{\circ} \mathrm{C}$ in Tokyo and $31^{\circ} \mathrm{C}$ and $35^{\circ} \mathrm{C}$ in Nanjing and Wuhan, respectively.

(2) Increasing surface temperatures could expand the regions in which the incidence of vector-borne and water-borne disease is very high. The spread of these diseases into more northern and southern latitudes would be an indirect impact on human health of climate change. Currently the highest incidences of these diseases are confined to tropical regions of the world. Of particular concerm are the re-emergence in areas that have been free for a long time from malaria and dengue fever as vector-borne diseases and from cholera as a water-borne disease. Increased temperatures (especially minimum temperatures) can increase the range of vector-borne diseases, particularly in regions where minimum temperatures may currently limit pathogen and/or vector development.

(3) Increasing surface temperatures could result in a greater frequency of heat waves and heat related mortality, primarily in urban areas. Over $65 \%$ of carbon dioxide emissions are from the combustion of fossil fuels, and increasing atmospheric concentraions of carbon dioxide could result in increases in both higher temperatures and air pollutant concentrations. Exposures could be to a combination of higher temperatures and higher air pollution concentrations. Therefore, it is important to estimate to what extent increasing air pollutant concentrations will contribute to mortality. Using time series analysis of data from Los Angeles for 1970 to 1979, regression formulas were developed for cardiovascular and respiratory mortality that were quadratic functions of temperature and linear functions of pollutant concentration. A second moment method was used to evaluate the contribution of air pollution to mortality for different age groups and different places where people live in Los Angeles. It was shown that as temperature and air pollutant concentrations increase, the contribution of air pollution to mortaliry increases.

(4) In the northem region of Argentina near the borders with Brazil and Paraguay, new cases of malaria occur most often during the months of March and April, with a few or no new cases occumng during the rest of the year. Starting in 1990 , this epidemic-type of occurrence changed into an endemictype occurrence, with cases occurring during almost the entire year, until 1993. It was suggested that the greater frequency and duration of El Nino-Southern Oscillation (ENSO) events, a large Pacific Ocean climatic system, may be causing longer periods of time when temperatures are favorable for the survival of malaria mosquitoes and parasites. Data from several meteorological stations in northern Argentina for 4 decades, indicate that average daily high temperatures have not changed much, but that average daily minimum temperatures have been gradually increasing between $0.4^{\circ} \mathrm{C}$ and $1.6^{\circ} \mathrm{C}$. At the same time, the seasonal activity of some important vectors has changed, with activity being observed during almost the entire year. Minimum temperatures for parasite survival and mosquito flying are $15.6^{\circ} \mathrm{C}$ and $20.0^{\circ} \mathrm{C}$ respectively. These temperatures are not as important for another important parasitic and heart pathology disease, Chagas' disease, because the most important vectors are domestic hemiptera and therefore are less affected by extemal meteorological conditions. Higher temperatures may be important for extending the geographical distribution of wild vectors, but may also accelerate the life cycle or affect population dynamics of those insects living indoors.

\section{COMMENTS}

It was suggested that there is a complex interaction between social and cultural responses to climate change. These interactions and their impacts on human health will need to be considered in the continuing efforts to assess the impacts of global climate change on human health. Agricultural crop failures in many developing countries can force people to migrate to sections of large urban areas in which sanitaion is poor and malnutrition high.

With regard to the regression formulas for cardiovascular and respiratory mortaliry for the city of Los Angeles, it was suggested that additional research was required to determine if correlations with these same general functional characteristics for temperature and air pollutant concentrations as were observed for Los Angeles, existed for other large metropolitan areas worldwide.

It was suggested that research should continue to find reliable biological indicators of heat stress that would make it possible to identify people who would be at greater risk for heat stress related mortality and morbidity. 